\title{
Beneficial Effect of Coenzyme Q10 on Increased Oxidative and Nitrative Stress and Inflammation and Individual Metabolic Components Developing in a Rat Model of Metabolic Syndrome
}

\author{
Masaru Kunitomo ${ }^{1, *}$, Yu Yamaguchi ${ }^{1}$, Satomi Kagota ${ }^{1}$, and Kazumasa Otsubo ${ }^{2}$ \\ ${ }^{1}$ School of Pharmaceutical Sciences, Mukogawa Women's University, Nishinomiya, Hyogo 663-8179, Japan \\ ${ }^{2}$ Fine Chemicals Division, Asahi Kasei Pharma Corporation, Tokyo 101-8101, Japan
}

Received December 15, 2007; Accepted April 11, 2008

\begin{abstract}
Metabolic syndrome (MetS) is a group of cardiovascular risk factors, including visceral obesity, glucose intolerance, hypertension, and dyslipidemia. Increased oxidative and nitrative stress and inflammation and decreased endothelial function occur in an animal model of metabolic syndrome, SHR/NDmcr-cp (SHR/cp) rats. The present study investigated the effects of coenzyme Q10 (CoQ10), one of the important antioxidants, on the abnormal oxidative condition and characteristic components of metabolic syndrome in SHR/cp rats by maintaining them on a diet supplemented with $0.07 \%-0.7 \%$ CoQ10 for 26 weeks. We determined serum levels of oxidatively modified low-density lipoprotein (Ox-LDL) and 8-hydroxy-2'-deoxyguanosine $(8-\mathrm{OHdG})$ as oxidative stress markers, 3-nitrotyrosine as a nitrative stress marker, 3chlorotyrosine as a marker of myeloperoxidase (MPO)-catalyzed oxidation and high-sensitivity $\mathrm{C}$-reactive protein (hsCRP) as an inflammatory marker. The administration of CoQ10 significantly attenuated the increase of oxidative and nitrative stress markers and inflammatory markers in a dose-dependent manner. CoQ10 prevented the elevated serum insulin levels, although it did not affect the elevated glucose level and dyslipidemia. CoQ10 also reduced elevated blood pressure, but did not affect body weight gain. In addition, CoQ10 improved endothelial dysfunction in the mesenteric arteries. These findings suggest that the antioxidant properties of CoQ10 can be effective for ameliorating cardiovascular risk in MetS.
\end{abstract}

Keywords: coenzyme Q10, metabolic syndrome model rat, oxidative stress, inflammation, endothelial dysfunction

\section{Introduction}

Metabolic syndrome (MetS) is a clinical feature characterized by a cluster of classic cardiovascular risk factors, including hypertension, hyperglycemia, and dyslipidemia (1). Visceral obesity and insulin resistance are currently considered primary factors underlying the development of the syndrome $(2,3)$. It has been reported that the proinflammatory state of visceral obesity induces insulin resistance, leading to clinical and biochemical manifestations of MetS $(4,5)$. Furthermore, systemic oxidative stress is associated with insulin

*Corresponding author. masaru@mukogawa-u.ac.jp Published online in J-STAGE on June 5, 2008 (in advance) doi: $10.1254 /$ jphs.FP0072365 resistance, visceral fat accumulation, and MetS $(6-8)$.

Coenzyme Q10 (CoQ10) is an endogenously synthesized compound that acts as an electron carrier in the mitochondrial respiratory chain (9). In addition to its unique role in mitochondria, CoQ10 functions as an antioxidant, scavenging free radicals and inhibiting lipid peroxidation $(10,11)$. Recent studies have provided evidence of the potential value of CoQ10 in prophylaxis and therapy of various disorders related to oxidative stress. There is promising evidence of the beneficial effect of CoQ10 in hypertension and heart failure $(12-14)$. It has been reported that CoQ10 concentrations and redox status are associated with components of MetS (15). Thus, it would be important to establish whether exogenous administration of CoQ10 is effective for prophylaxis and therapy of MetS. 
SHR/NDmcr-cp/cp (SHR/cp) rats manifest hypertension (derived from SHR), obesity (as a result of a nonsense mutation in the leptin-receptor gene), glucose intolerance, and hyperlipidemia (16). They have been used as an animal model of MetS $(16-18)$. We have previously reported that oxidative and nitrative stress, together with inflammation are systemically elevated with the development of metabolic disorders characteristic to SHR/cp rats (19).

In the present study, we investigated the effect of exogenous CoQ10 administration on the increase of oxidative and nitrative stress markers and inflammatory markers and the individual components of MetS by using SHR/cp rats. We also examined the effect of CoQ10 on the vascular endothelial dysfunction developing in these rats.

\section{Materials and Methods}

\section{Materials}

Chemicals of analytical reagent grade were purchased from Nacalai Tesque (Kyoto) and used without further purification. The oxidized form of CoQ10 was kindly donated by Asahi Kasei Pharma Co. (Tokyo). ProtEXDEAE columns (particle diameter of $5 \mu \mathrm{m}$, column size: $50 \mathrm{~mm} \times 4.6 \mathrm{~mm}$ i.d.), which were used for anionexchange high-performance liquid chromatographic (AE-HPLC) assay, were kindly donated by Mitsubishi Chemical Co. (Tokyo). Water purified with a Milli Q Jr. (Millipore, Tokyo) was used to prepare eluents for the HPLC method.

\section{Animals}

The SHR/cp rat is a subline of the spontaneously hypertensive/NIH-corpulent (SHR/N-cp) rat distributed by the National Institutes of Health (Bethesda, MD, USA), which was established under specific pathogenfree (SPF) conditions by the Disease Model Cooperative Research Association (Kyoto). Six-week-old male SHR/cp rats were purchased from Japan SLC, Inc. (Hamamatsu). The rats were bred with a high-fat diet consisting of $14 \%$ fat, $46 \%$ carbohydrate, and $24 \%$ protein (CLEA Rodent Diet Quick Fat; CLEA Japan, Inc., Tokyo), which is used for an animal model of type 2 diabetes mellitus and obesity. As shown in Table 1, the rats were divided into control, $0.07 \%$ CoQ10, $0.2 \%$ CoQ10, and $0.7 \%$ CoQ10 groups, which were fed the diet supplemented with $0 \%, 0.07 \%$, $0.2 \%$ and $0.7 \%$ CoQ 10 , respectively, for 26 weeks. At 20 weeks of age, the middle period of the experiment, the body weight of each group was $617 \pm 15,647 \pm 16$, $639 \pm 17$, and $663 \pm 17 \mathrm{~g}$ and the mean food consumption was $23.4,24.0,23.5$, and $23.8 \mathrm{~g}$, respectively. Thus the supplementation of CoQ10 to each group was approximately equivalent to dosages of $0,26,74$, and $252 \mathrm{mg} / \mathrm{kg}$ per day, respectively. Both the diets and water were given ad libitum during the experimental period. There were no significant differences among the diet intakes of the four groups throughout the experiment. All rats were kept in an air-conditioned room $\left(23 \pm 2^{\circ} \mathrm{C}\right.$ and $55 \pm 10 \%$ humidity) under an artificial 12 h light/dark cycle (07:00 - 19:00). The systolic blood pressure and heart rate were determined at 4-week intervals with conscious rats from 6 weeks of age by the indirect tail-cuff method. The measurement of blood pressure was stopped at 22 weeks of age because the blood pressure of the SHR/cp rats became too elevated (over $200 \mathrm{mmHg}$ ). The study protocols were performed according to the Guideline Principles for the Care and Use of Laboratory Animals approved by The Japanese Pharmacological Society.

\section{Preparation of serum samples}

After overnight fasting of the rats, blood was drawn from the tail vein of 6-week-old animals and thereafter at 4-week intervals from 8 weeks of age under light anesthesia with diethyl ether, and it was drawn from the abdominal aorta at the end of the experiment (32 weeks of age) with the animals under anesthesia with pentobarbital sodium $(40 \mathrm{mg} / \mathrm{kg}$, i.p.). The serum was separated by centrifugation $(1,500 \times g$ for $10 \mathrm{~min})$ from the clotted blood, stored at $-80^{\circ} \mathrm{C}$ and then used for measurement of various lipids, high-density lipoprotein (HDL), low-density lipoprotein (LDL), very low-density lipoprotein (VLDL), subfractions of LDL, glucose, insulin, 3-nitrotyrosine, 3-chlorotyrosine, 8-hydroxy-2'deoxyguanine (8-OHdG), and high-sensitivity C-reactive protein (hsCRP).

\section{Determination of CoQ10}

The serum levels of CoQ10 (Ubiquinol-10 and ubiquinone-10) were simultaneously determined using HPLC with electrochemical detection (HPLC-ECD), as described by Yamashita and Yamamoto (20), except that extraction in hexane and methanol was replaced by the use of isopropanol.

\section{Determination of oxidatively modified $L D L$}

An anion-exchange HPLC method was used to measure the oxidatively modified LDL (Ox-LDL) in the serum, according to a previously described method (21, 22). Serum LDL was separated by stepwise elution into three subfractions: LDL1, LDL2, and LDL3. The cholesterol level of each subfraction was detected using an enzymatic post-column reaction and evaluated as a relative value to the total cholesterol levels in LDL. The 
degree of oxidative modification of serum LDL was assessed by the decrease in LDL1 and the increase in LDL2 and LDL3. Thus, LDL1, 2, and 3 refer to normal, mildly modified, and severely modified fractions, respectively.

\section{Determination of $8-O H d G$}

The HPLC-ECD method was used to measure 8OHdG in the serum, according to a method described by Long et al. (23). Briefly, an aliquot of serum was extracted by addition of methanol. After centrifugation at $7000 \times g$ for $10 \mathrm{~min}$, the supernatant was dried under a stream of nitrogen gas. The residues were dissolved in the mobile phase solution in this HPLC method. The dissolved sample was centrifuged again at $7,000 \times g$ for $10 \mathrm{~min}$, and an aliquot of the supernatant was injected into the HPLC-ECD system. The voltage of the guard cell was set at $500 \mathrm{mV}$, and those of the first and second electrodes of the analytical cell set at 150 and $300 \mathrm{mV}$, respectively. The analytical column was a Cosmosil 5C18-AR-II (particle diameter, $5 \mu \mathrm{m}$; column size, $150 \times 4.6 \mathrm{~mm}$ I.D.) equipped with a guard column $(10 \times 4.6 \mathrm{~mm} \mathrm{I.D.})$, and the mobile phase was $20 \mathrm{mM}$ sodium phosphate buffer, $\mathrm{pH} 4.7$, including $10 \%(\mathrm{v} / \mathrm{v})$ methanol. The flow rate was $1 \mathrm{~mL} / \mathrm{min}$ and the column temperature was set at $25^{\circ} \mathrm{C}$. Quantitation of $8-\mathrm{OHdG}$ was performed by comparison of the peak area with that of authentic 8-OHdG.

\section{Determination of 3-nitrotyrosine and 3-chlorotyrosine}

The HPLC-ECD method was used to measure 3nitrotyrosine and 3-chlorotyrosine in the total serum proteins, according to a previously described method (22). Briefly, the lipid in an aliquot of serum was extracted with ethanol/ether $(3: 1, \mathrm{v} / \mathrm{v})$, and the residue containing total protein was hydrolyzed with $6 \mathrm{M}$ hydrochloric acid for $12 \mathrm{~h}$ at $110^{\circ} \mathrm{C}$. After removal of hydrochloric acid from the hydrolysate, the residue was dissolved in distilled water, and an aliquot of the supernatant was injected into the HPLC-ECD system. The voltage of the guard cell was set at $800 \mathrm{mV}$ and those of the first and second electrodes of the analytical cell were set at 550 and at $750 \mathrm{mV}$, respectively. The analytical column was the Cosmosil 5C18-AR-II equipped with the guard column described above and the mobile phase was $20 \mathrm{mM}$ sodium phosphate buffer, $\mathrm{pH} 3.0$, including $5 \%(\mathrm{v} / \mathrm{v})$ methanol. The flow rate was $1 \mathrm{~mL} / \mathrm{min}$ and the column temperature was set at $20^{\circ} \mathrm{C}$. Quantitation of 3-nitrotyrosine and 3-chlorotyrosine was performed by comparison of the peak areas with those of authentic 3-nitrotyrosine and 3-chlorotyrosine, respectively.

\section{Relaxation studies}

At the end of the experiment ( 32 weeks of age), the mesenteric artery was removed and immediately placed in Krebs-Henseleit solution $(118.4 \mathrm{mM} \mathrm{NaCl}, 4.7 \mathrm{mM}$ $\mathrm{KCl}, 2.5 \mathrm{mM} \mathrm{CaCl}_{2}, 1.2 \mathrm{mM} \mathrm{KH}_{2} \mathrm{PO}_{4}, 1.2 \mathrm{mM} \mathrm{MgSO}_{4}$, $25.0 \mathrm{mM} \mathrm{NaHCO}_{3}$, and $11.1 \mathrm{mM}$ glucose). The vessels were cut into $3-\mathrm{mm}$ rings, and the rings were mounted isometrically at an optimal resting tension of $0.3 \mathrm{~g}$ in a $10-\mathrm{mL}$ organ bath filled with the solution $\left(37^{\circ} \mathrm{C}, \mathrm{pH} 7.4\right)$ described above. The bath solution was continuously aerated with a gas mixture of $95 \% \mathrm{O}_{2}$ and $5 \% \mathrm{CO}_{2}$. Isometric tension change was measured with a forcedisplacement transducer (Model t-7; NEC San-Ei, Tokyo) coupled to a dual channel chart recorder (Model $8 \mathrm{~K} 21$, NEC San-Ei). The rings were preconstricted with phenylephrine $(1-3 \mu \mathrm{M})$ to generate approximately $80 \%$ of the maximal contraction. Once a stable contraction was obtained, acetylcholine was cumulatively added to the bath. The relaxation response obtained was expressed as a percentage of the maximal relaxation developed by papaverine $(100 \mu \mathrm{M})$.

\section{Biochemical analysis}

Serum total cholesterol levels were determined by a fluoro-enzymatic method as described previously (24). HDL, LDL, and VLDL levels were determined by an AE-HPLC method as described previously (25). Triglyceride, free fatty acid, and glucose levels were evaluated by Triglyceride E test Wako, NEFA C test Wako, and Glucose C II test Wako (Wako Pure Chemical Industries, Osaka), respectively. Serum insulin levels were measured with a Rat Insulin ELISA Kit (Morinaga Institute of Biological Science, Inc., Kanagawa). Serum hsCRP levels were measured with a Rat C-Reactive Protein ELISA Kit (Alpha Diagnostic International, San Antonio, TX, USA). Protein levels in non-lipid serum were measured by using a BCA protein assay kit (Pierce, Rockford, IL, USA).

\section{Statistics}

The results are each expressed as the mean \pm S.E.M. Individual concentration-response curves for acetylcholine were characterized to determine the $\mathrm{pEC}_{50}$ and $R_{\max }$, which were calculated using GraphPad Prism software (Ver. 4; San Diego, CA, USA). Statistical analyses were performed by analysis of variance (ANOVA) followed by the Bonferroni multiple comparison test and Bonferroni-Dunn test (Stat View software, Ver. 5.0; SAS, Cary, NC, USA). A difference was considered significant when $P<0.05$. 


\section{Results}

Changes in body weight and serum CoQ10 levels

Table 1 shows the body weight of each group on the initial and final days of the experiment, at 6 and 32 weeks of age, respectively. There were no significant differences in body weight among the groups throughout the experiments.

As shown in Table 2, serum levels of CoQ10, mostly ubiquinol, were markedly increased by administration of CoQ10 in a dose-related manner throughout the experiment; a marked increase after the two-week administration followed by a gradual increase until the end of experiments.

\section{Effect of CoQ10 on heart rate and blood pressure}

As shown in Fig. 1, the initial systolic blood pressure was similar for all groups. The systolic blood pressure in the control group became elevated quite rapidly with age. The blood pressures of the CoQ10-treated groups were significantly lower than that of the control group after 18 weeks of age. There were no significant differences in the heart rate among all groups.
Effect of CoQ10 on serum insulin and glucose levels

The serum glucose and insulin levels of SHR/cp rats were markedly higher than those of normal Wistar Kyoto rats (19). CoQ10 administration did not affect the serum glucose levels in the control group throughout the experimental period (Fig. 2A). In contrast, the CoQ10treated groups showed a significant decrease in the serum insulin levels compared to the control group in a dose-related manner after 16 weeks of age and the suppressive effects continued until the end of the experiment (Fig. 2B).

\section{Effect of CoQ10 on serum lipid levels}

The lipid and lipoprotein levels of SHR/cp rats were markedly higher than those of Wistar Kyoto rats, except for HDL-cholesterol (19). Figure 3 shows the changes in the serum total cholesterol (Fig. 3A) and triglyceride (Fig. 3B) levels in each group. CoQ10 administration did not affect these levels throughout the experimental period. Similarly, CoQ10 administration did not affect the increased levels of free fatty acids, LDL-cholesterol and VLDL-cholesterol, and the decreased levels of HDL-cholesterol in the control group (data not shown).

Table 1. Grouping and changes in body weight

\begin{tabular}{llccc}
\hline \multirow{2}{*}{ Group } & Diet & \multirow{2}{*}{ No. of rats } & \multicolumn{2}{c}{ Body weight (g) } \\
\cline { 3 - 5 } & & & Initial & Final \\
\hline Control & Quick Fat & 6 & $185 \pm 7$ & $843 \pm 20$ \\
$0.07 \%$ CoQ10 & Quick Fat supplemented with $0.07 \%$ CoQ10 & 6 & $184 \pm 8$ & $852 \pm 22$ \\
$0.2 \%$ CoQ10 & Quick Fat supplemented with $0.2 \%$ CoQ10 & 6 & $183 \pm 7$ & $844 \pm 24$ \\
$0.7 \%$ CoQ10 & Quick Fat supplemented with $0.7 \%$ CoQ10 & 6 & $184 \pm 9$ & $877 \pm 25$ \\
\hline
\end{tabular}

Each value represents the mean \pm S.E.M. for 6 rats. CoQ10, coenzyme Q10.

Table 2. Serum CoQ10 (ubiquinol and ubiquinone) levels in SHR/cp rats administered exogenous CoQ10

\begin{tabular}{lcccc}
\hline \multirow{2}{*}{ Group } & $\begin{array}{c}\text { Age } \\
\text { (weeks) }\end{array}$ & Total & Ubiquinol & Ubiquinone \\
\cline { 3 - 4 } Control & 8 & & $\mathrm{nM}$ & \\
& 32 & $23 \pm 2$ & $23 \pm 2$ & N.D. \\
$0.07 \%$ CoQ10 & 8 & N.D. & N.D. & N.D. \\
& 32 & $515 \pm 27$ & $491 \pm 25$ & $24 \pm 3$ \\
$0.2 \%$ CoQ10 & 8 & $470 \pm 62$ & $445 \pm 61$ & $24 \pm 2$ \\
& 32 & $707 \pm 88$ & $677 \pm 84$ & $30 \pm 4$ \\
$0.7 \%$ CoQ10 & 8 & $1020 \pm 233$ & $986 \pm 232$ & $34 \pm 4$ \\
& 32 & $1012 \pm 39$ & $971 \pm 38$ & $41 \pm 2$ \\
\hline
\end{tabular}

Exogenous ubiquinone was administered to SHR/cp rats from 6 weeks of age. Each value represents the mean \pm S.E.M. for 6 rats. CoQ10, coenzyme Q10; N.D., not detected. 

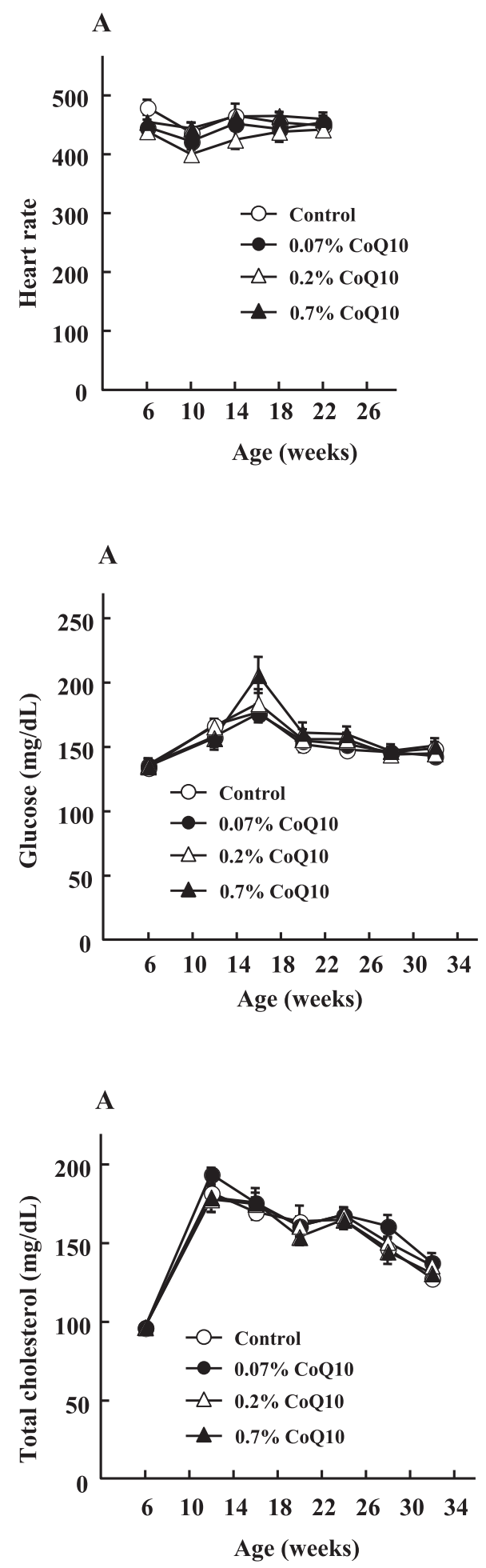
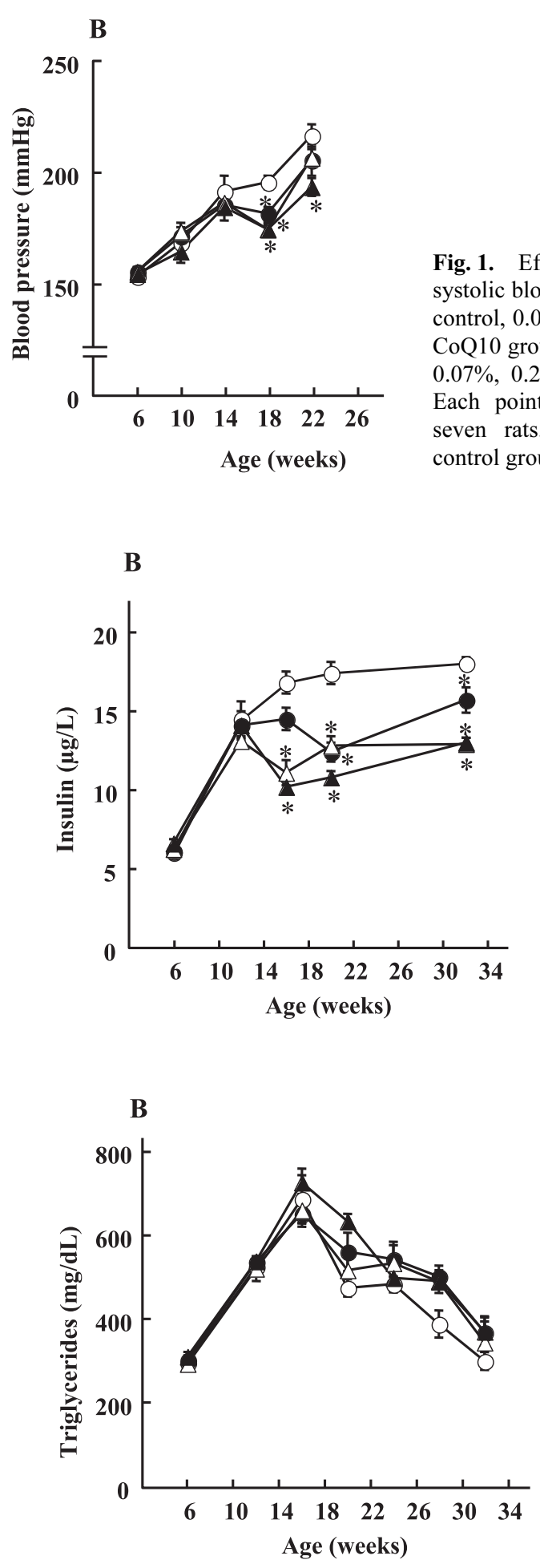

Fig. 1. Effects of CoQ10 on heart rate (A) and systolic blood pressure (B) in SHR/cp rats. The control, $0.07 \%$ CoQ10, $0.2 \%$ CoQ10, and $0.7 \%$ CoQ10 groups were fed the diet containing $0 \%$, $0.07 \%, 0.2 \%$, and $0.7 \%$ CoQ10, respectively. Each point represents the mean \pm S.E.M. of seven rats. Significant difference from the control group, ${ }^{*} P<0.05$.
Fig. 2. Effects of CoQ10 on glucose (A) and insulin (B) levels in the serum of SHR/cp rats. The control, $\quad 0.07 \% \quad$ CoQ10, $0.2 \%$ CoQ10, and $0.7 \%$ CoQ10 groups were fed the diet containing $0 \%$, $0.07 \%, 0.2 \%$, and $0.7 \%$ CoQ 10 , respectively. Each point represents the mean \pm S.E.M. of seven rats. Significant difference from the control group, $* P<0.05$.

Fig. 3. Effects of CoQ10 on total cholesterol (A) and triglyceride (B) levels in the serum of $\mathrm{SHR} / \mathrm{cp}$ rats. The control, $0.07 \%$ CoQ10, $0.2 \%$ CoQ10, and $0.7 \%$ CoQ10 groups were fed the diet containing $0 \%$, $0.07 \%, 0.2 \%$, and $0.7 \%$ CoQ 10 , respectively. Each point represents the mean \pm S.E.M. of seven rats.

Effect of CoQ10 on biomarker levels of oxidative stress

Ox-LDL and 8-OHdG were measured as biomarkers of oxidative stress. Figure 4 shows the relative levels of LDL subfractions, LDL1, LDL2, and LDL3 (Fig. 4: A, $\mathrm{B}$, and $\mathrm{C}$, respectively). In the control group, the levels of LDL1 significantly decreased while those of LDL2 and LDL3, especially severely modified LDL3, significantly increased. CoQ10 administration significantly reversed the LDL1 and LDL3 levels in a dose-related manner. The alteration of LDL2 levels was not sufficient but similar to that of LDL3 levels.

SHR/cp rats display higher 8-OHdG levels compared 

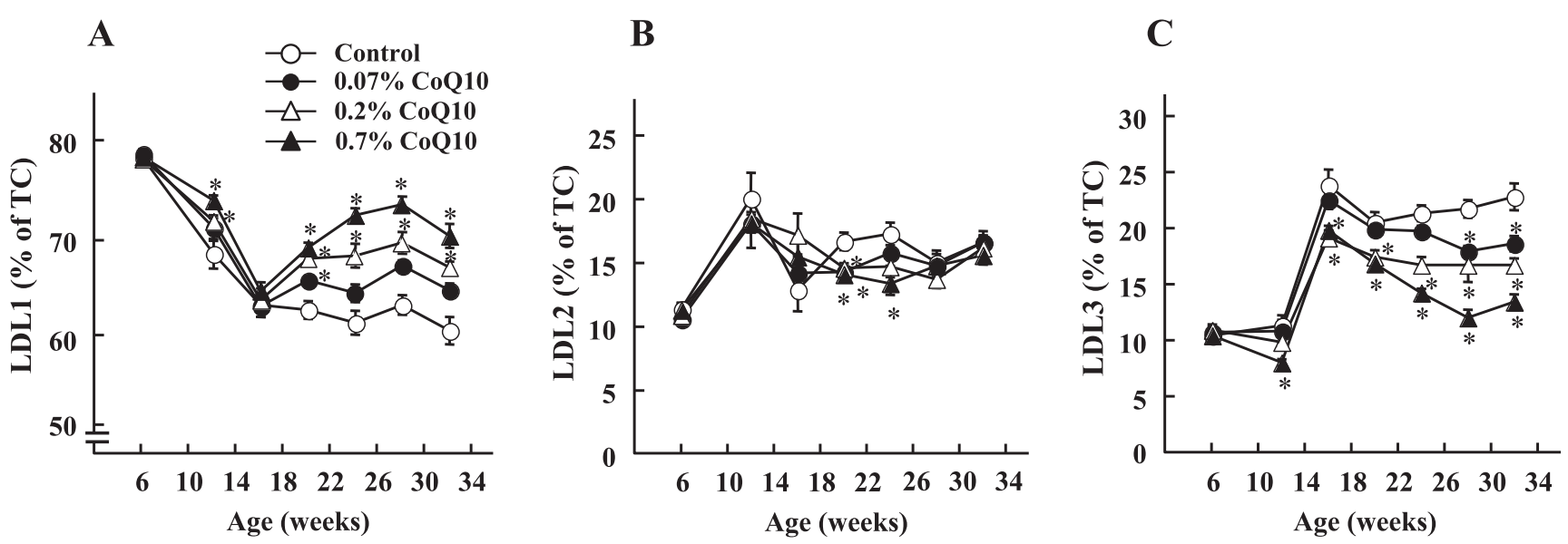

Fig. 4. Effects of CoQ10 on the ratios of LDL subfractions, LDL1 (A), LDL2 (B), and LDL3 (C) in SHR/cp rats. The LDL subfractions were separated by an anion-exchange HPLC method with stepwise elution, as described in Materials and Methods. The control, $0.07 \%$ CoQ10, $0.2 \%$ CoQ10, and $0.7 \%$ CoQ10 groups were fed the diet containing $0 \%, 0.07 \%, 0.2 \%$, and $0.7 \%$ $\mathrm{CoQ} 10$, respectively. Each point represents the mean \pm S.E.M. of seven rats. Significant difference from the control group, $* P<0.05$. TC, total cholesterol.

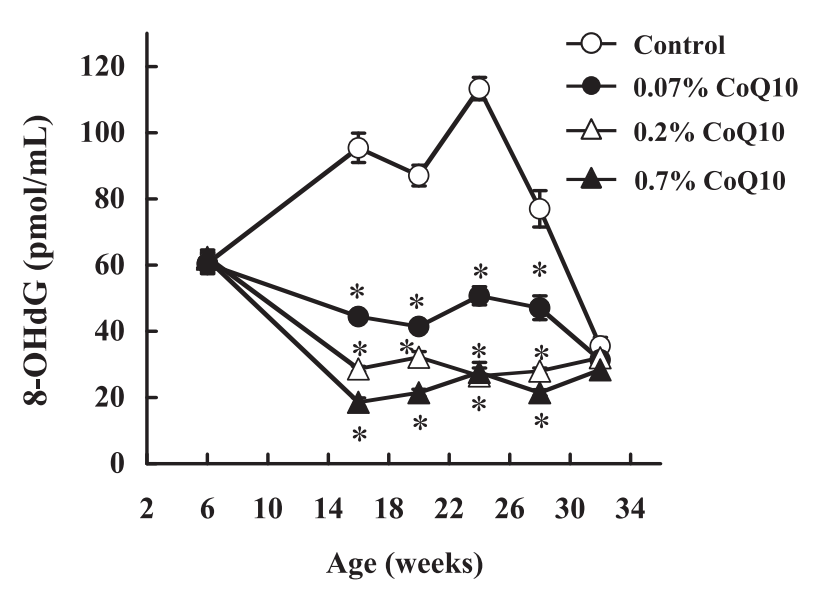

Fig. 5. Effects of CoQ10 on 8-hydroxy-2'-deoxyguanine (8-OHdG) levels in the serum of SHR/cp rats. The control, $0.07 \% \mathrm{CoQ} 10,0.2 \%$ CoQ10, and $0.7 \%$ CoQ10 groups were fed the diet containing $0 \%$, $0.07 \%, 0.2 \%$, and $0.7 \%$ CoQ10, respectively. Each point represents the mean \pm S.E.M. of seven rats. Significant difference from the control group, ${ }^{*} P<0.05$.

to Wistar Kyoto rats from 6 weeks of age (19). As shown in Fig. 5, CoQ10 administration dose-dependently decreased the serum 8-OHdG levels in the control group throughout the experimental period.

\section{Effect of CoQ10 on serum 3-nitrotyrosine and 3-chloro- tyrosine levels}

Figure 6 shows the levels of 3-nitrotyrosine (Fig. 6A) and 3-chlorotyrosine (Fig. 6B), a specific biomarker of peroxynitrite and myeloperoxidase (MPO)-catalyzed oxidation, respectively, in the serum total proteins. Although all groups showed normal levels of 3-nitro- tyrosine and 3-chlorotyrosine at 6 weeks of age, both levels in the control group gradually and significantly increased after 16 weeks of age, reached a peak at 20 weeks, and thereafter remained at markedly higher levels until 32 weeks of age. CoQ10-treated groups showed a significant decrease in both levels in a doserelated manner after 20 weeks of age until the end of the experiment.

\section{Effect of CoQ10 on serum hsCRP levels}

The serum levels of hsCRP, a sensitive marker of low-grade systemic inflammation, are shown in Fig. 7. The serum hsCRP level in the control group markedly increased after 16 weeks of age, reached a peak at 20 weeks of age, and gradually returned to the initial levels at the end of the experiment. The increased levels at 16 and 20 weeks of age were significantly suppressed by CoQ10 administration.

\section{Effect of CoQ10 on endothelium-dependent relaxation of mesenteric artery}

The relaxation in response to acetylcholine in the aorta of SHR/cp rats is markedly impaired compared with that in normal Wistar-Kyoto rats, as previously described (18). Figure 8 shows the acetylcholineinduced endothelium-dependent relaxation in the mesenteric artery from the control and $0.7 \%$ CoQ10 groups at the end of the experiment. The acetylcholineinduced relaxation response was not sufficient in the control group, but was approximately $100 \%$ in the $0.7 \%$ CoQ10 group, which was equivalent to the endothelial function of the normal rats. 

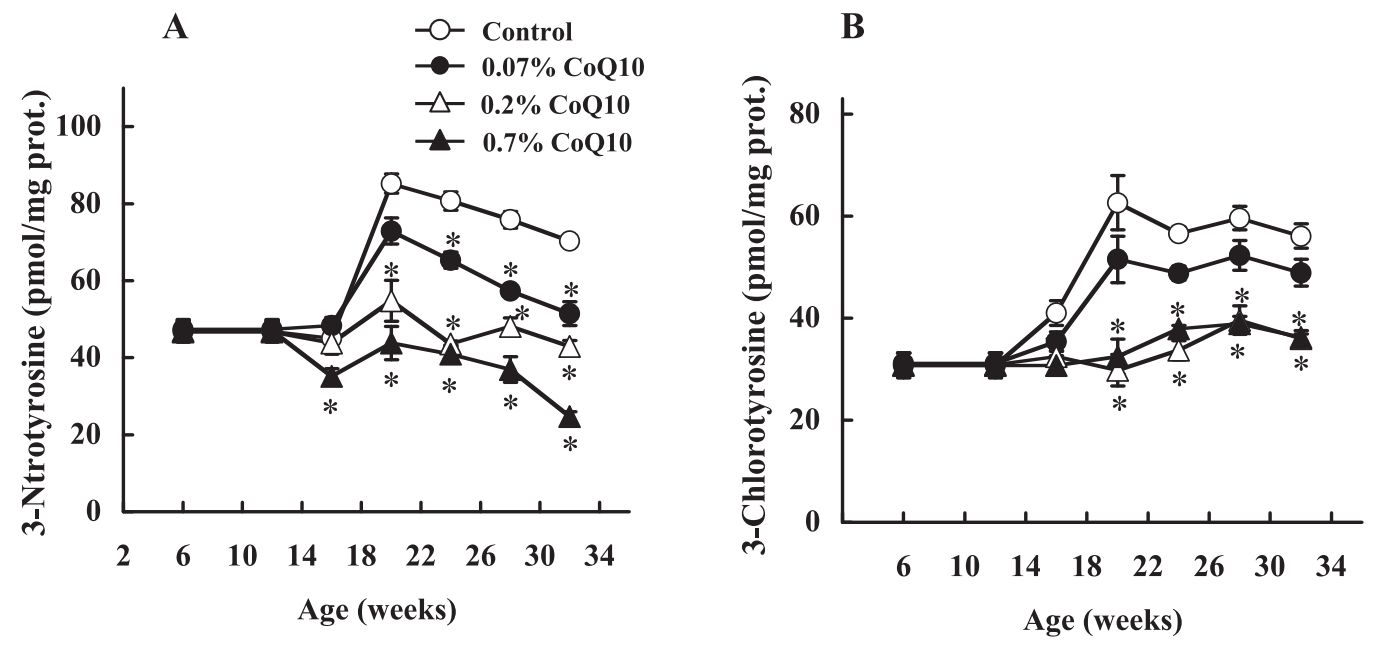

Fig. 6. Effects of CoQ10 on 3-nitrotyrosine (A) and 3-chlorotyrosine (B) levels in the serum protein of SHR/cp rats. The control, $0.07 \% \mathrm{CoQ} 10,0.2 \% \mathrm{CoQ} 10$, and $0.7 \% \mathrm{CoQ} 10$ groups were fed the diet containing $0 \%, 0.07 \%, 0.2 \%$, and $0.7 \% \mathrm{CoQ} 10$, respectively. Each point represents the mean \pm S.E.M. of seven rats. Significant difference from the control group, ${ }^{*} P<0.05$.

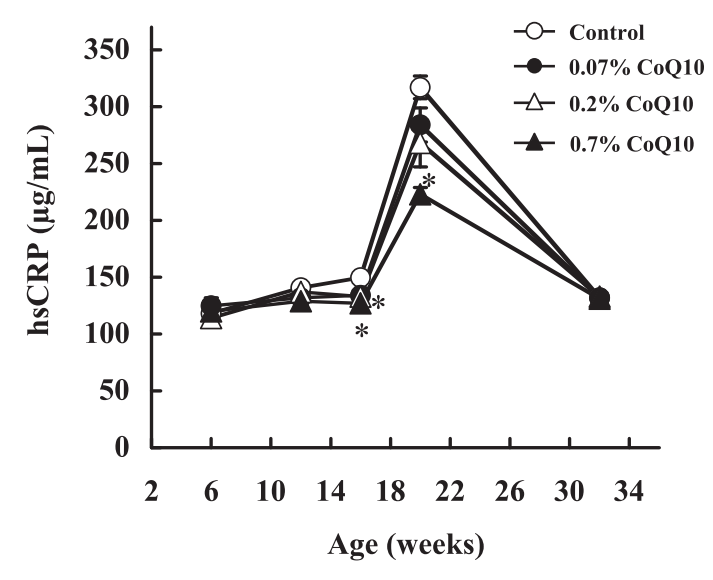

Fig. 7. Effects of CoQ10 on serum high-sensitivity C-reactive protein (hsCRP) levels of SHR/cp rats. The control, $0.07 \%$ CoQ10, $0.2 \%$ CoQ10, and $0.7 \%$ CoQ 10 groups were fed the diet containing $0 \%, 0.07 \%, 0.2 \%$, and $0.7 \%$ CoQ 10 , respectively. Each point represents the mean \pm S.E.M. of seven rats. Significant difference from the control group, ${ }^{*} P<0.05$.

\section{Discussion}

Oxidative stress, overproduction of reactive oxygen species, has a critical role in the pathogenesis of endothelial dysfunction and atherosclerosis (26-29). We have previously demonstrated that systemic oxidative stress and nitrative stress as well as inflammation increase with the development of MetS-like components in SHR/cp rats (19), which display abdominal obesity, hypertension, hyperglycemia, insulin-resistance, and hyperlipidemia $(16,18)$. Several clinical studies have provided evidence that systemic oxidative stress, as measured by urinary 8-epi-prostaglandin $F_{2 \alpha}$ (6) or

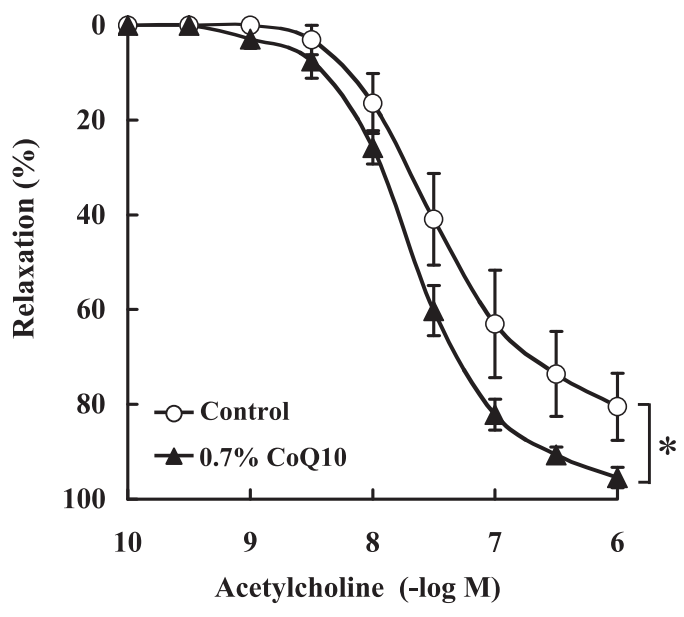

Fig. 8. Effects of CoQ10 on endothelium-dependent relaxations in response to acetylcholine in the mesenteric artery of SHR/cp rats. The relaxation response was expressed as a percentage of the maximal relaxation caused by papaverine $(100 \mu \mathrm{M})$. The control and $0.7 \%$ CoQ10 groups were fed the diet containing $0 \%$ and $0.7 \%$ CoQ10, respectively. Statistical analysis was performed using the analysis of variance (ANOVA) followed by the Bonferroni-Dunn test for the $\mathrm{pEC}_{50}$ and $R_{\max }$ values. Significant difference from the control group, ${ }^{*} P<0.05$.

plasma Ox-LDL $(7,8)$, is associated with MetS. To prevent the oxidative damage to the vascular endothelium that is observed in the early stage of atherosclerosis $(14,30)$, the antioxidant CoQ10 is considered to be a promising therapeutic agent. However, there have been no studies on the effect of CoQ10 on the pathologic condition of MetS. In the present study, we demonstrated for the first time that long-term CoQ10 administration can prevent increased oxidative and nitrative 
stress, as indicated by higher levels of Ox-LDL and 8$\mathrm{OHdG}$ in the serum and of 3-nitrotyrosine in serum proteins, respectively, and the increased inflammation with activation of MPO, as indicated by higher serum levels of hsCRP and 3-chlorotyrosine in the SHR/cp rats displaying MetS-like components. In addition, we found that the elevated serum insulin levels and high blood pressure were suppressed by CoQ10 intake. These improvement effects were clearly and dose-dependently manifested after treatment with CoQ10 for 10 weeks. Furthermore, we found that CoQ10 ameliorates endothelial dysfunction in the mesenteric arteries of the rats. However, CoQ10 administration did not affect the abnormal weight gain, dyslipidemia (elevated levels of total cholesterol, triglycerides, free fatty acids, LDL- and VLDL-cholesterol, and reduced HDL-cholesterol), and hyperglycemia developing in the serum of the SHR/cp rats.

When the oxidized form of CoQ10 (ubiquinone) was orally administered to SHR/cp rats, CoQ10 in the serum was mostly present in the reduced form, ubiquinol, after 2 weeks. It has been reported that a high dose of CoQ10 at $150 \mathrm{mg} / \mathrm{kg}$ per day increases the CoQ10 content in the liver, kidney, heart, skeletal muscle, brain, and mitochondria of these tissues of rats (31). Therefore, the dosages of CoQ10 used in the present study, $25-$ $250 \mathrm{mg} / \mathrm{kg}$ per day, seem to show a sufficient antioxidant effect in various tissues. Indeed, we found that the oxidative and nitrative stress markers were reduced in a dose-related manner, although it took about 10 weeks for CoQ10 administration to display a significant effect. These findings indicate that CoQ10 levels seem to increase rapidly in the serum but slowly in the tissues.

Ox-LDL, an oxidative stress marker is also widely regarded as a critical factor in the atherogenic process (32). Circulating Ox-LDL is also consistently associated with the pro-inflammatory cytokine tumor necrosis factor $\alpha$ and CRP (33). 8 -OHdG is also a reliable marker for the assessment of oxidative DNA damage and vascular oxidative stress (34). Increased oxidative stress is correlated with enhanced systemic inflammation (35). In the present study, we found that CoQ10 administration decreases both Ox-LDL and 8-OHdG levels in the serum. This finding may be linked to the alleviation of inflammation and endothelial dysfunction. Indeed, CoQ10 administration diminished the increased level of hsCRP, a sensitive systemic marker of inflammation, in the serum of SHR/cp rats after 16 weeks of age, although the effect was transient. Elevated levels of hsCRP are known to be an important prognostic predictor of coronary risk (36). In SHR/cp rats, therefore, oxidative stress may affect hsCRP levels and promote vascular inflammatory processes.

MPO is an abundant hemoprotein that is released by activated neutrophils, monocytes, and tissue-associated macrophages after inflammatory stimulation. MPO catalyzes a reaction between chloride and hydrogen peroxide to produce hypochlorous acid, which reacts with tyrosine residues of proteins to form 3-chlorotyrosine (37). In vivo, MPO can also produce 3-nitrotyrosine (38), which is widely recognized as an inflammation-associated marker that is formed by nitration of protein tyrosine residues with peroxynitrite $\left(\mathrm{ONOO}^{-}\right)$ generated during inflammation. A variety of inflammatory cells produce nitric oxide (NO) and superoxide $\left(\mathrm{O}_{2}^{-}\right)$, yielding $\mathrm{ONOO}^{-}(39)$. Thus, the increased 3chlorotyrosine and 3-nitrotyrosine levels in the serum of $\mathrm{SHR} / \mathrm{cp}$ rats are considered to result from MPO activation and/or $\mathrm{ONOO}^{-}$production via an inflammatory response. Taken together, our findings suggest that the antioxidative effect of CoQ10 can act against a series of inflammatory processes that develop in SHR/cp rats displaying a MetS-like pathologic condition.

These findings suggest that the antioxidative and antiinflammatory effects of CoQ10 can help prevent the development of atherosclerosis. Unfortunately, rats are generally resistant to the development of atherosclerosis (40, 41). As expected, we could not histologically observe atherosclerotic injury in the aorta and arteries of $\mathrm{SHR} / \mathrm{cp}$ rats. Alternatively, we found that CoQ10 administration ameliorates the reduced endotheliumdependent relaxation induced by acetylcholine in the mesenteric arterial ring preparation of SHR/cp rats. Inflammation and oxidative stress have been demonstrated to be associated with endothelial dysfunction in subjects with MetS (42) and animal models of disease $(29,43)$. We have also previously reported that endothelium-dependent relaxations are impaired in the aorta of SHR/cp rats due to peroxynitrite generated in the vessel walls $(18,44)$. We think that NADPH oxidasederived superoxide, probably produced due to stimulation of $\mathrm{AT}_{1}$ receptors, reacts with $\mathrm{NO}$ to form peroxynitrite, which decreases active NO and damages endothelial cells, leading to attenuation of endotheliumdependent relaxation. These findings indicate that CoQ10 can reverse endothelial dysfunction by preventing oxidative and nitrative stress and inflammation.

Endothelial dysfunction and increased oxidative stress are characteristics of patients with essential hypertension. As superoxide anion reduces NO bioavailability and acts as a vasoconstrictor $(45,46)$, endothelial dysfunction can lead to increased peripheral resistance and high blood pressure. Thus, our findings that CoQ10 prevents vascular endothelial dysfunction seem to be linked to its hypotensive effect in SHR/cp rats. Further- 
more, insulin resistance and the consequent hyperinsulinemia, important components of MetS, are associated with endothelial dysfunction (47), probably due to increasing oxidative stress $(48,49)$. The physiological properties of insulin that cause enhancement of renal sodium reabsorption and stimulate sympathetic nervous system activity are believed to play a major role in the development of hypertension (50), although the underlying mechanisms in the setting of insulin resistance remain obscure (51). Therefore, the hypotensive effect of CoQ10 observed in SHR/cp rats may be associated with its alleviation of hyperinsulinemia together with endothelial dysfunction.

In conclusion, we demonstrated that administration of CoQ10 notably suppresses oxidative and nitrative stress, inflammation, hypertension, and hyperinsulinemia developing in SHR/cp rats, a model of MetS. These findings suggest that the antioxidant properties of CoQ10 can be effective for ameliorating cardiovascular risk in MetS.

\section{Acknowledgements}

This work was supported in part by a grant from the Smoking Research Foundation; a Grant-in-Aid for Scientific Research from the Ministry of Education, Culture, Sports, Science, and Technology of Japan (16590100, to M.K.); and also by the Open Research Center Project of Mukogawa Women's University for studying lifestyle-related diseases.

\section{References}

1 Grundy SM, Cleeman JI, Daniels SR, Donato KA, Eckel RH, Franklin BA, et al. Diagnosis and management of the metabolic syndrome: an American Heart Association/National Heart, Lung, and Blood Institute Scientific Statement. Circulation. 2005;112:2735-2752.

2 Reaven GM. Metabolic syndrome: pathophysiology and implications for management of cardiovascular disease. Circulation. 2002;206:286-288

3 Cheal KL, Abbasi F, Lamendola C, McLaughlin T, Reaven GM, Ford ES. Relationship to insulin resistance of the adult treatment panel III diagnostic criteria for identification of the metabolic syndrome. Diabetes. 2004;53:1195-1200.

4 Dandona P, Aljada A, Chaudhuri A, Mohanty P, Garg R. Metabolic syndrome: a comprehensive perspective based on interactions between obesity, diabetes, and inflammation. Circulation. 2005;111:1448-1454.

5 Shoelson SE, Herrero L, Naaz A. Obesity, inflammation, and insulin resistance. Gastroenterology. 2007;132:2169-2180.

6 Fujita K, Nishizawa H, Funahashi T, Shimomura I, Shimabukuro M. Systemic oxidative stress is associated with visceral fat accumulation and the metabolic syndrome. Circ J. 2006;70:1437-1442.
7 Sigurdardottir V, Fagerberg B, Hulthe J. Circulating oxidized low-density lipoprotein (LDL) is associated with risk factors of the metabolic syndrome and LDL size in clinically healthy 58year-old men (AIR study). J Intern Med. 2002;252:440-447.

8 Lapointe A, Couillard C, Piche ME, Weisnagel SJ, Bergeron J, Nadeau A, et al. Circulating oxidized LDL is associated with parameters of the metabolic syndrome in postmenopausal women. Atherosclerosis. 2007;191:362-368.

9 Crane FL, Hatefi Y, Lester RL, Widmer C. Isolation of a quinone from beef heart mitochondria. Biochim Biophys Acta. 1957;25:220-221.

10 Frei B, Kim MC, Ames BN. Ubiquinol-10 is an effective lipidsoluble antioxidant at physiological concentrations. Proc Natl Acad Sci U S A. 1990;87:4879-4883.

11 Ernster L, Dallner G. Biochemical, physiological and medical aspects of ubiquinone function. Biochim Biophys Acta. 1995; 1271:195-204.

12 Rosenfeldt FL, Haas SJ, Krum H, Hadj A, Ng K, Leong JY, et al. Coenzyme Q10 in the treatment of hypertension: a metaanalysis of the clinical trials. J Hum Hypertens. 2007;21:297306.

13 Pepe S, Marasco SF, Haas SJ, Sheeran FL, Krum H, Rosenfeldt FL. Coenzyme Q10 in cardiovascular disease. Mitochondrion. 2007;7 Suppl:S154-S167.

14 Singh U, Devaraj S, Jialal I. Coenzyme Q10 supplementation and heart failure. Nutr Rev. 2007;65:286-293.

15 Miles MV, Morrison JA, Horn PS, Tang PH, Pesce AJ. Coenzyme Q10 changes are associated with metabolic syndrome. Clin Chim Acta. 2004;344:173-179.

16 Nagase M, Yoshida S, Shibata S, Nagase T, Gotoda T, Ando K, et al. Enhanced aldosterone signaling in the early nephropathy of rats with metabolic syndrome: possible contribution of fatderived factors. J Am Soc Nephrol. 2006; 17:3438-3446.

17 Velliquette RA, Kossover R, Previs SF, Ernsberger P. Lipidlowering actions of imidazoline antihypertensive agents in metabolic syndrome X. Naunyn Schmiedebergs Arch Pharmacol. 2006;372:300-312.

18 Kagota S, Yamaguchi Y, Tanaka N, Kubota Y, Kobayashi K, Nejime N, et al. Disturbances in nitric oxide/cyclic guanosine monophosphate system in SHR/NDmcr-cp rats, a model of metabolic syndrome. Life Sci. 2006;78:1187-1196.

19 Yamaguchi Y, Yoshikawa N, Kagota S, Nakamura K, Haginaka J, Kunitomo M. Elevated circulating levels of markers of oxidative-nitrative stress and inflammation in a genetic rat model of metabolic syndrome. Nitric Oxide. 2006;15:380-386.

20 Yamashita S, Yamamoto Y. Simultaneous detection of ubiquinol and ubiquinone in human plasma as a marker of oxidative stress. Anal Biochem. 1997;250:66-73.

21 Yamaguchi Y, Kagota S, Kunitomo M, Haginaka J. Evidence of modified lipoprotein in the plasma of Watanabe Heritable Hyperlipidaemic rabbits by anion-exchange high-performance liquid chromatographic assay. Atherosclerosis. 1998;139:323331.

22 Yamaguchi Y, Kagota S, Haginaka J, Kunitomo M. Participation of peroxynitrite in oxidative modification of LDL by aqueous extracts of cigarette smoke. FEBS Lett. 2002;512:218-222.

23 Long L, Mccabe DR, Dolan ME. Determination of 8-oxoguanine in human plasma and urine by high-performance liquid chromatography with electrochemical detection. J Chromatgr B. 1999;731:241-249. 
24 Kunitomo M, Yamaguchi Y, Matsushima K, Bando Y. Cholesterol metabolism in serum and aorta of inbred mice fed a high-cholesterol diet. Jpn J Pharmacol. 1984;34:153-158.

25 Yamaguchi Y, Kunitomo M, Haginaka J. Anion-exchange highperformance liquid chromatographic assay of plasma lipoproteins of rabbits, rats and mice. J Chromatogr B Biomed Sci Appl. 1998;716:57-64.

26 Witztum JL. The oxidation hypothesis of atherosclerosis. Lancet. 1994;344:793-795.

27 Harrison D, Griendling KK, Landmesser U, Hornig B, Drexler $\mathrm{H}$. Role of oxidative stress in atherosclerosis. Am J Cardiol. 2003;91:7A-11A.

28 Madamanchi NR, Vendrov A, Runge MS. Oxidative stress and vascular disease. Arterioscler Thromb Vasc Biol. 2005;25:2938 .

29 Nyby MD, Abedi K, Smutko V, Eslami P, Tuck ML. Vascular angiotensin type 1 receptor expression is associated with vascular dysfunction, oxidative stress and inflammation in fructose-fed rats. Hypertens Res. 2007;30:451-457.

30 Kaliora AC, Dedoussis GV, Schmidt H. Dietary antioxidants in preventing atherogenesis. Atherosclerosis. 2006;187:1-17.

31 Kwong LK, Kamzalov S, Rebrin I, Bayne AC, Jana CK, Morris $\mathrm{P}$, et al. Effects of coenzyme Q(10) administration on its tissue concentrations, mitochondrial oxidant generation, and oxidative stress in the rat. Free Radic Biol Med. 2002;33:627-638.

32 Witztum JL, Steinberg D. Role of oxidized low density lipoprotein in atherogenesis. J Clin Invest. 1991;88:1785-1792.

33 Hulthe J, Fagerberg B. Circulating oxidized LDL is associated with subclinical atherosclerosis development and inflammatory cytokines (AIR Study). Arterioscler Thromb Vasc Biol. 2002; 22:1162-1167.

34 Floyd RA, West MS, Eneff KL, Schneider JE, Wong PK, Tingey DT, et al. Conditions influencing yield and analysis of 8-hydroxy-2'-deoxyguanosine in oxidatively damaged DNA. Anal Biochem. 1990;188:155-158.

35 Somfai GM, Knippel B, Ruzicska E, Stadler K, Toth M, Salacz $\mathrm{G}$, et al. Soluble semicarbazide-sensitive amine oxidase (SSAO) activity is related to oxidative stress and subchronic inflammation in streptozotocin-induced diabetic rats. Neurochem Int. 2006;48:746-752.

36 Libby P, Ridker PM. Novel inflammatory markers of coronary risk: theory versus practice. Circulaton. 1999;100:1148-1150.

37 Himmelfarb J, McMenamin E, Loseto G, Heinecke JW. Myeloperoxidase-catalyzed 3-chlorotyrosine formation in dialysis patients. Free Radic Biol Med. 2001;31:1163-1169.

38 Gaut JP, Byun J, Tran HD, Lauber WM, Carroll JA, Hotchkiss
RS, et al. Myeloperoxidase produces nitrating oxidants in vivo. J Clin Invest. 2002;109:1311-1319.

39 Shigenaga MK, Lee HH, Blount BC, Christen S, Shigeno ET, Yip $\mathrm{H}$, et al. Inflammation and NOx-induced nitration: assay for 3-nitrotyrosine by HPLC with electrochemical detection. Proc Natl Acad Sci U S A. 1997;94:3211-3216.

40 Moghadasian MH. Experimental atherosclerosis: a historical overview. Life Sci. 2002;70:855-865.

41 Bartuś M, Lomnicka M, Lorkowska B, Franczyk M, Kostogrys RB, Pisulewski PM, et al. Hypertriglyceridemia but not hypercholesterolemia induces endothelial dysfunction in the rat. Pharmacol Rep. 2005;57 Suppl:127-137.

42 Chapman MJ. Metabolic syndrome and type 2 diabetes: lipid and physiological consequences. Diab Vasc Dis Res. 2007;4 Suppl 3:S5-S8.

43 Cai H, Harrison DG. Endothelial dysfunction in cardiovascular diseases: the role of oxidant stress. Circ Res. 2000;87:840-844.

44 Kagota S, Tada Y, Kubota Y, Nejime N, Yamaguchi Y, Nakamura K, et al. Peroxynitrite is involved in the dysfunction of vasorelaxation in SHR/NDmcr-cp rats, spontaneously hypertensive obese rats. J Cardiovas Pharmacol. 2007;50:677685.

45 Ferroni P, Basili S, Paoletti V, Davi G. Endothelial dysfunction and oxidative stress in arterial hypertension. Nutr Metab Cardiovasc Dis. 2006;16:222-233.

46 Taddei S, Virdis A, Mattei P, Salvetti A. Vasodilation to acetylcholine in primary and secondary forms of human hypertension. Hypertension. 1993;21:929-933.

47 Steinberg HO, Chaker H, Leaming R, Johnson A, Brechtel $\mathrm{G}$, Baron AD. Obesity/insulin resistance is associated with endothelial dysfunction: implications for the syndrome of insulin resistance. J Clin Invest. 1996;97:2601-2610.

48 Arcaro G, Cretti A, Balzano S, Lechi A, Muggeo M, Bonora E, et al. Insulin causes endothelial dysfunction in humans: sites and mechanisms. Circulation. 2002;105:576-582.

49 Picchi A, Gao X, Belmadani S, Potter BJ, Focardi M, Chilian $\mathrm{WM}$, et al. Tumor necrosis factor-alpha induces endothelial dysfunction in the prediabetic metabolic syndrome. Circ Res. 2006;99:69-77.

50 Ginsberg HN. Insulin resistance and cardiovascular disease. J Clin Invest. 2000;106:629-631

51 Ceriello A, Motz E. Is oxidative stress the pathogenic mechanism underlying insulin resistance, diabetes and cardiovascular disease? The common soil hypothesis revisited. Arterioscler Thromb Vasc Biol. 2004;24:816-823. 